\title{
Internet Protocol Support for Telephony
}

\author{
MASSIMO MARESCA, NICOLA ZINGIRIAN, AND PIERPAOLO BAGLIETTO, MEMBER, IEEE
}

\author{
Invited Paper
}

In this paper, we consider the evolution of telephone networks from time-division multiplexing circuit switching to packet switching and, in particular, to packet switching-based on Internet Protocol (IP-supported telephony). We analyze IP-supported telephony design solutions by proposing a layered reference model in which each layer is associated to a subset of the functions that support telephony. We use the reference model to establish a terminology and a framework for the comparison of the design solutions. We group the design solutions in scenarios and compare them in terms of the reference model proposed. We then focus on IP telephony, in which IP is used in telephone company networks, and on Internet telephony, in which the Internet is used to support telephony. We show that they both can be seen as implementations of the same architecture, which consists of a set of components, associated to functions, and of the interactions among these components. We then consider the issue of voice-data integration and analyze the variety of design solutions that can be adopted to integrate voice and data.

Keywords-Internet Protocol (IP) telephony, Internet telephony, next-generation networks, voice over Internet Protocol (VoIP).

\section{NOMENCLATURE}

ATM Asynchronous transfer mode, a cell-switched communications technology.

CB Customer base (legacy dimension).

CP Content provider.

CS Circuit switching.

DE Dedicated equipment (legacy dimension).

DNS Domain name system.

DSL Digital subscriber line.

DT Dedicated technology (legacy dimension).

DTMF Dual-tone multiple frequency.

E.164 An ITU-T standard for telephone numbering plan.

ENUM IETF standard for the mapping of telephone numbering on DNS.

Manuscript received December 9, 2003; revised March 26, 2004.

M. Maresca and N. Zingirian are with the Department of Information Engineering, University of Padova, Padova 35131, Italy (e-mail: massimo.maresca@unipd.it).

P. Baglietto is with the Department of Communication, Computer and Systems Engineering, University of Genova, Genova 13-16145, Italy.

Digital Object Identifier 10.1109/JPROC.2004.832950
G.711 An ITU-T standard for voice coding.

H.323 An ITU-T standard protocol suite for real-time communication over a packet network.

IETF Internet Engineering Task Force.

IP Internet Protocol.

IPSec IP Security.

ISDN Integrated services digital network.

ISP Internet service provider.

ITU International Telecommunications Union.

MPLS Multiprotocol label switching.

OLO Other licensed operators: nonincumbent telephone companies.

P(A)BX Private (automatic) branch exchange, usually used on business premises to switch telephone calls.

PCM Pulse code modulation.

PDH Plesiochronous digital hierarchy.

PPP Point-to-point technology.

PS Packet switching.

PSTN Public switched telephone network.

Q.931 An ITU-T standard for user-side signaling.

RFC Request for comments.

RTP Real-time protocol.

SDH Synchronous digital hierarchy.

SIP Session initiation protocol.

SL Service level (legacy dimension).

SS7 Signaling system 7.

TA Terminal addressing (legacy dimension).

TCO Telephone company.

TDM Time-division multiplexing.

UI User interface (legacy dimension).

URI Uniform resource identifier.

VoIP Voice over IP.

\section{INTRODUCTION}

Telephone networks are rapidly evolving from TDM-CS technologies to PS technologies. This evolution is aimed at reducing the TCO costs associated to network implementation, operation, maintenance, and management, at making possible the fusion of voice and data networks over the same 
network, and at supporting the integration of voice and data in multimedia services. Network cost reduction is obtained by exploiting the statistical multiplexing capability offered by PS, which leads to a better utilization of the network resources, as well as by taking advantage of the low cost of PS equipment due to the widespread diffusion of Internet technologies. The fusion of voice and data networks over the same network is obtained, for those companies that operate both a data network and a telephone network, through the implementation of a common high-speed backbone, over which the data network and the telephone network can be implemented as virtual networks. The integration of voice and data in multimedia services is obtained through the possibility of treating voice as a data type, thus enabling the coordinated processing of voice and data.

IP has currently remained the only suitable protocol for PS networks, mainly because of the diffusion of the Internet, which has led to a significant reduction of the cost of IP equipment as well as to a significant growth of the number of technicians and engineers trained in Internet technologies. The fact that IP has no significant competitor has led to the idea of using IP to transport telephony. This is usually referred to as VoIP (see, for example, [16]). Specific architectures and protocols for VoIP were developed both in the ITU domain [20], [42] and in the IETF domain [17], [18] and in a joint domain [3]. These architectures and protocols have been validated in public telephone networks [33], in corporate telephone networks [30], and on the Internet [31].

However, there is a variety of solutions in which IP can be used to support telephony. These solutions, which we group under the expression "IP-supported telephony," are associated to two different approaches, which we call the "telephone philosophy" and the "Internet philosophy," having in common the use of IP as a PS protocol but aiming at different objectives.

In particular, according to the telephone philosophy, the introduction of IP in telephone service implementation is a strategy that TCOs may adopt to reduce their costs by replacing the existing Class 5 and Class 4 switches with lowcost IP routers and to enrich the set of services that they offer to their customers by using low-cost computer systems to implement and manage such services.

On the contrary, according to the Internet philosophy, the use of IP to transport voice is a strategy that users may follow to reduce telephone service costs and to stimulate application service providers to conceive, implement, and offer new multimedia services in which voice and data are processed in a coordinated way.

The difference between the two views is a new form in which the dispute between the Internet approach and the telephone approach appears. According to the telephone approach, the network is "intelligent" [14], to mention a typical adjective used in telephony, and plays a fundamental role in the implementation and in the management of application services. On the contrary, according to the Internet approach, the network is "stupid" [9] and takes care only of data transport, whereas the application services are implemented and managed at the network end points.
The analysis of the design solutions according to which IP can be used to support telephony is conducted in this paper by first proposing a reference model for IP-supported telephony which consists of a set of layers, each of which is associated to a set of functions to be performed to support telephony. The reference model is used to establish a terminology and a framework for the comparison of the different design solutions. We classify the solutions in four different scenarios and compare and discuss the four scenarios in terms of the reference model proposed. We then focus on IP telephony, in which the IP is used in TCO networks, and Internet telephony, in which the Internet is used to support telephony. We show that they both can be seen as implementations of the same architecture, which consists of a set of components, associated to functions, and of the interactions among these components. We present such an architecture and show how both IP telephony and Internet telephony are implementations of such an architecture, obtained by assigning different functions to the architecture components.

We then consider the issue of voice-data integration, which is one of the objectives that drive the transition of telephone networks from TDM-CS to IP. We consider the case in which a user has a connection to an IP network, typically the Internet, and a connection to the telephone network, and analyze the variety of design solutions that can be adopted to integrate the two services. We classify these solutions in four schemes that we present in detail. For each scheme we show if and how voice-data integration takes place.

The paper proceeds as follows. In Section II we describe and compare the philosophies of the telephone community and the Internet community which inspire the way in which IP-supported telephony is considered. In Section III we describe the telephone legacy and its influence on the evolution toward IP-supported telephony. In Section IV we propose the IP-supported telephony reference model. In Section V we present the common architecture of IP telephony and Internet telephony. In Section VI we present the voice-data integration schemes. Finally, in Sections VII and VIII, we first discuss the concepts presented, and then we provide a concluding remark.

\section{Telephone Philosophy Versus InTERNET PHILOSOPHY}

The fundamental element that makes the migration of telephony from TDM-CS to IP-PS a realistic objective is the fact that the evolution of IP technology has led to a point at which IP equipment and transmission lines are able to route and transport an IP traffic of the same size and of the same characteristics as the ones of telephone traffic at a satisfactory service level.

While many analyses and comparisons of Internet traffic and telephone traffic can be found in the literature (see, for example, [7] and [34]), a simple but concrete idea of how the Internet traffic has already overcome the telephone traffic can be given by looking at the Italian situation. Considering 
that the incumbent TCO (Telecom Italia) transports 4.5 billion min of voice conversations in one year between the two largest Italian cities, Rome and Milan [6], and assuming that all the traffic is concentrated in 200 working days, we obtain that the average telephone traffic exchanged corresponds to 15625 Erlangs (i.e., minutes of conversation per minute). Supposing to code each conversation using G.723.1 [21] at $12.8 \mathrm{~kb} / \mathrm{s}(6.4 \mathrm{~kb} / \mathrm{s}$ each way), the average data traffic intensity corresponds to $200 \mathrm{Mb} / \mathrm{s}$, and considering the overhead due to protocols (on the order of $100 \%$ due to $40 \mathrm{~B}$ for protocol headers for each 32-B payload corresponding to $40 \mathrm{~ms}$ of audio signal), the total traffic intensity to be taken into account is $400 \mathrm{Mb} / \mathrm{s}$. This traffic intensity is comparable with the intensity of the Internet traffic transported by a medium-size Italian ISP. As an example, we may take the Italian Research Network (GARR) [13] backbone that transports an average traffic of about $300 \mathrm{Mb} / \mathrm{s}$ between Rome and Milan.

Once the fact is established that IP equipment can be used to support the telephone service transport infrastructure, it may be worth investigating whether a migration of telephony from TDM-CS to IP-PS must be regarded only as a convenient technical solution to improve efficiency in existing telephone networks or whether such a migration may have implications also on other aspects of the telephone service, such as, for example, the service implementation architecture, the standardization process, and the service provisioning business model.

We start such an investigation by summarizing and comparing the principles that are at the basis of the telephone approach and of the Internet approach. In the rest of this section, we refer to the principles that have driven and drive the evolution of the telephone network as the "telephone philosophy," whereas we refer to the approaches that have driven and drive the development of the Internet as the "Internet philosophy."

1) Expensive Versus Cheap Technology: In the telephone philosophy, the technology involved is extremely complex and expensive; as a consequence, it requires huge investments for equipment deployment so that only public or very large companies can purchase and manage a telephone infrastructure. The telephone infrastructure is based on systems that are located in a few centers, properly presided, and are operated and managed by one or a few organizations. On the contrary, in the Internet philosophy, the Internet infrastructure is based on simple and cheap systems [5] that can be operated and managed by smaller size companies (ISPs). It is the coordinated action of many small or medium-sized companies, rather than of a few large companies, that has determined and determines the growth of the Internet.

2) Bureaucratic Versus Agile Standardization: In the telephone philosophy, the definition of a standard is similar to the definition of an international treaty (de jure standard), which requires a long time and a large effort both to set up an agreement among the committee components and to capture all the technical details in the standard definition documents. On the contrary, in the Internet philosophy, the standardization mechanism is very agile, being that the spec- ifications are minimal and the standard acceptance is based on experimental demonstrations and on "rough consensus." An evident example of agile standardization can be found in the historical document that defines the core of the Internet standard [35], i.e., the IP protocol: here the author, Postel, after proposing a trivial mechanism for error checking in the IP packet header, inserted the following remark: "This is a simple [way] to compute checksum and experimental evidence indicates it is adequate, but it is provisional and may be replaced by a [cyclic redundancy code] procedure, depending on further experience." At the present time, about 25 years later, such a "provisional" algorithm is effectively adopted on millions of IP nodes.

3) Application Service Versus Transport Service: In the telephone philosophy, the telephone service customers are not considered "network users" while they are considered "telephone service users," as TCOs offer end-to-end voice transmission services and hide the network services. On the contrary, in the Internet philosophy, users can access the network at the transport level and build up their own application services at the endpoints, independently of the ISPs.

4) Centralized Service Versus Distributed Service: In the telephone philosophy, the core network supports the base application service (i.e., the telephone call) as well as the additional services (e.g., voice mail, redirection, etc.), as all the applications are implemented inside the network. On the contrary, in the Internet philosophy, the core network is not supposed to implement applications, which means that the services are necessarily implemented at the network periphery.

5) Monopolistic Versus Pluralistic Service Provisioning: In the telephone philosophy, customers cannot subscribe to services other than the ones provided by the TCO that provides the base service (i.e., telephony). On the contrary, in the Internet philosophy, users are encouraged to purchase application services from a variety of application service providers, using the Internet only as a way to transport these services.

6) Regulated Versus Spontaneous Business: In the telephone philosophy, the telephone service is an essential facility, in the same way as water or electricity distribution. As a consequence, the telephone service business is controlled by appropriate organizations - in some cases directly by the state governments - to guarantee the so-called universal service [40], i.e., the service provisioning at affordable rates for all the users independently of the actual interconnection costs. On the contrary, in the Internet philosophy, the growth of the network is prevalently driven by free market rules.

7) Commercial Versus Noncommercial Interprovider Agreement: In the telephone philosophy, the telephone service business model is based on the precise accounting of the telephone traffic, call by call. When a TCO exchanges traffic with another TCO, it considers each incoming call as a service provided to the other company (and accounts the duration of that call as a revenue), whereas it considers each outgoing call as a service received from the other company (and accounts the duration of that call as a cost to be redirected to the customers). On the contrary, in the Internet 
philosophy, interprovider relationships are based on a rough estimation of the IP aggregated traffic and, in many cases, on noncommercial agreements. If an ISP provides connectivity to another ISP toward other networks or toward the rest of the Internet, it sells this service through a "transit" contract whose entity depends on the traffic volume exchanged. If two ISPs exchange traffic with each other only to interconnect their own customers with a direct link and no other networks, they establish a noncommercial agreement called "peering." The point is that an ISP stipulates one or a few transit contracts, usually with international carriers (tier-1 ISPs), and hundreds peering agreement with other ISPs [43].

8) Rigid Versus Flexible Resource Allocation: In the telephone philosophy, the resource elements to be allocated are the "lines," which correspond to fixed bandwidth (i.e., $64 \mathrm{~kb} / \mathrm{s}$ ) circuits and are hardwired in systems based on TDM. As a consequence, the planning of the capacity of a telephone exchange is typically a part of the design phase, impacts on the number of units to be installed, and is driven by well-known rigid rules that guarantee the availability of lines with a given probability. On the contrary, in the Internet philosophy, the resource elements to be allocated are the capacities of the links carrying aggregated traffic (e.g., measured in gigabits per second) and the capacities of switching/routing equipment (e.g., measured in million frames or packets per second). As soon as the traffic is about to saturate the capacity of links or switches or routers, the network manager usually upgrades the resources immediately.

9) Guaranteed Service Versus Best Effort: In the telephone philosophy, the service is supposed to be provided at high quality. Quality refers both to transmission parameters (e.g., ITU MOS for perceived quality) and to availability (e.g., the "five nines" requirement). On the contrary, in the Internet philosophy, the service is provided at best effort quality, both in transmission and in availability.

10) Volume-Based Versus Flat Charges: In the telephone philosophy, the telephone call is the service charged to the customer. Except for the local calls, typically the telephone calls are charged taking into account the conversation duration and the distance covered. This charge model discourages the utilization of long distance lines and allows the TCOs to keep the number of such lines small with respect to the number of users attached. On the contrary, in the Internet philosophy, the connection to the Internet is the service charged to the customer, independently of the connection time and of the distance covered, and the pricing model is prevalently flat. This model encourages the network utilization and, in the worst cases, i.e., in event of link saturation, the service level decreases.

\section{TELEPHONE LEGACY DimENSIONS}

More than one century of telephony has consolidated probably the most significant legacy system ever installed in the world. The significance of telephone legacy is not only due to its impressive size-e.g., in terms of cost of equipment and lines involved, number of users connected, amount of turnovers, and revenues for TCOs [10] — but also to a variety of subtle aspects, which we call "legacy dimensions" and describe in this section.

1) UI: Generations of users have so far accessed the telephone service through a well-known interaction paradigm with terminals. Actions such as hanging up or dialing and conventional signals such as ringing or free/busy tones are universally accepted.

2) TA: Telephone networks take advantage of a worldwide numbering system [22] to identify the telephone users. The acceptance of this type of addressing is demonstrated by the fact that no transparent name-to-number resolution has ever been provided by TCOs.

3) $S L$ : Telephone service has raised the expectation of high levels of availability [29] and quality [38], [1] in generations of users. At the moment, the fact that one could receive an unclear voice signal or find the line busy or silent just after picking up the phone does not belong to the common feeling of telephone users.

4) DT: Telephone network technologies rigidly satisfy architectural assumptions that, in some cases, do not hold any more. The persistence of such assumptions makes the telephone infrastructures unable to take advantage of innovative technologies. For instance, one of the most limiting assumptions is the correspondence between a telephone conversation and a $64 \mathrm{~kb} / \mathrm{s}$ switched circuit. Well-known voice coding algorithms have made such an assumption no longer valid, compressing voice streams at a bit rate of 13 $\mathrm{kb} / \mathrm{s}$ [11] or $6.4 \mathrm{~kb} / \mathrm{s}$ [21]. Unfortunately such algorithms are inapplicable to the telephone network, since current TDM-CS telephone network equipment (e.g., switches and multiplexers) and standards (e.g., PDH) support neither switched circuits having a bit rate less than $64 \mathrm{~kb} / \mathrm{s}$ nor variable bit rate channels. This lack of flexibility keeps the telephone network resource utilization up to one order of magnitude larger than necessary.

5) $D E$ : The telephone network relies on equipment dedicated to voice relay (e.g., Class 5 and Class 4 switches). The downsizing phenomenon evolved remarkably more slowly for telephone equipment than for computer equipment, thus keeping the current market of telephone switches similar to the one of mainframes a couple of decades ago (see, for example, [32]).

6) $C B$ : TCOs, in particular the incumbent operators, have consolidated a huge $\mathrm{CB}$ that accepts to devolve a roughly constant budget to the telephone service, in spite of the general reduction of telecommunication costs. The existence of this legacy is demonstrated by the fact that in many countries public or independent entities, on behalf of governments, have undertaken actions aimed at checking the congruity of telephone rates with actual telecommunication costs.

All these dimensions play a crucial role in the convergence of IP networks with telephone networks. 


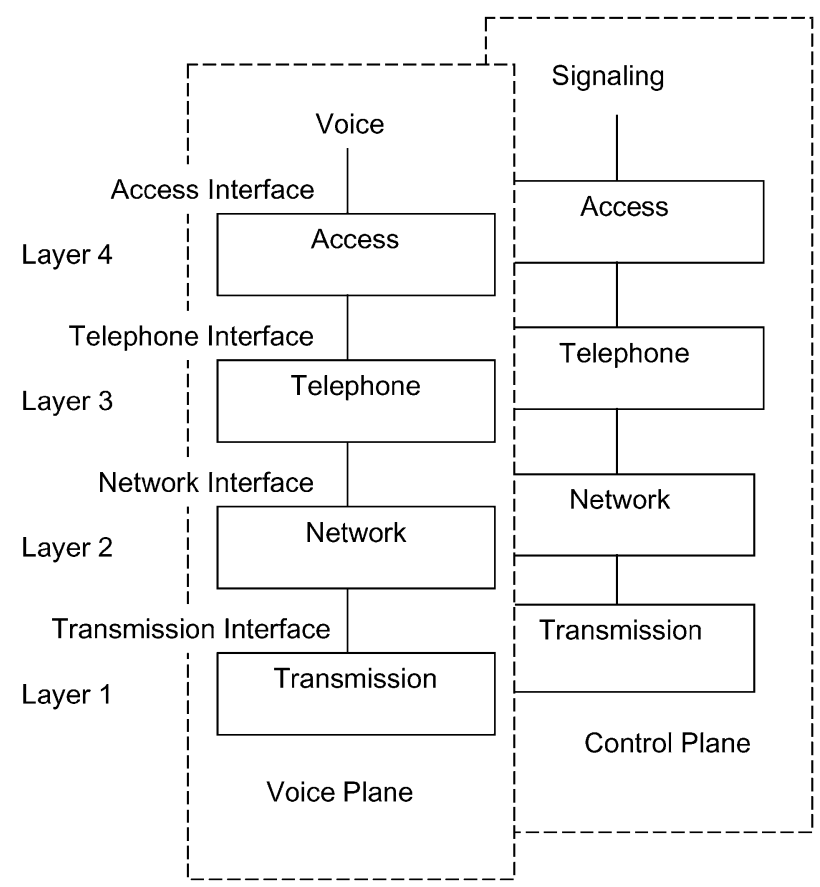

Fig. 1. IP-supported telephony reference model.

\section{IP-SUPPORTED TELEPHONY REFERENCE MODEL}

In this section we introduce a reference model for IP-supported telephony. We then describe the layers at which IP can be used to support telephony in terms of such a reference model. The possibility of using IP at different layers leads to the identification of four scenarios, which will be described in detail. While the first of these scenarios takes advantage of IP only to emulate trunks, the other three scenarios take advantage of IP for call control and transport. We associate the VoIP acronym only to these latter scenarios, as the VoIP protocols and technology (e.g., H.323 [20], SIP [18]) have as a specific function the support of call control and transport.

The proposed IP-supported telephony reference model includes: 1) four layers, namely, transmission, network, telephone, and access; 2) four interfaces, namely, transmission interface, network interface, telephone interface, and access interface; and 3) two planes, namely, the voice plane and the signaling plane, as shown in Fig. 1. Each layer includes the functions that allow mapping its interface to the higher level layer on the interface to the lower level layer. The two planes introduced-namely, the voice plane and the Signaling plane, respectively-refer to voice transmission and to call control.

Layer 1, i.e., the transmission layer, corresponds to the so-called IP core network of the TCO, assumed that a core IP Network is used. The transmission layer interface consists of point-to-point circuits implemented as emulated constant bit rate lines (e.g., T1/E1 [24]) or as variable bit rate IP tunnels (e.g., IPSEC [28]).

Layer 2, i.e., the network layer, corresponds to the network that supports the telephone service and is either a traditional PSTN/ISDN network built over the circuits provided by the transmission layer or an IP network built over the tunnels provided by the transmission layer. The network layer interface is different depending on whether the network layer is implemented as an IP network or using TDM-CS. In the first case, the network layer interface is the usual IP interface consisting of the primitives for sending/receiving datagrams over the network address space, while in the second case, the network layer interface consists of the standard interface for network signaling (e.g., SS7 [26]) and for digitized voice transmission (e.g., DS0 [24]).

Layer 3, i.e., the telephone layer, corresponds to the telephone service as it is presented to the user. The telephone layer interface consists of two interfaces, one for signaling and one for voice transmission, and may appear either as a regular signaling/transmission interface for PSTN/ISDN access (e.g., ISDN BRI [19], Q.931 [23]) or as an IP-based signaling/transmission interface (e.g., RTP [41], H.323).

Layer 4 , i.e., the access layer, corresponds to the functions that must be implemented in the user telephone set to access the telephone service under the control of the access layer interface. The access layer interface is the actual interface presented to the telephone user, such as, for example, the standard telephone set keyboard or a window/mouse-based interface for PC.

The reference model captures a variety of existing scenarios, which we describe in the rest of this section and summarize in Table 1.

The scenarios are described in the sections that follow.

\section{A. Scenario I-IP Trunking}

Scenario I illustrates the typical architecture of a traditional TCO that adopts an IP infrastructure instead of traditional long distance links or, equivalently, a private organization that replaces trunks (e.g., E1/T1) between remote PABXs with IP-based connections.

The access interface consists of the UI of the traditional telephone set. In the signaling plane, it corresponds to the 12-button keypad, busy/ringing tones, etc., and takes advantage of the global telephone numbering plan E.164 [22], while in the voice plane, it corresponds to the receiver speaker and microphone.

The access layer is implemented in the telephone set. In the signaling plane, it consists of user-side signaling generation/processing (e.g., DTMF generation and Q.931 information exchanges). In the voice plane, it consists of the transduction of voice to electrical signals in the case of analog telephone and of the coding of sampled voice (e.g., G.711 PCM [27]).

The telephone interface consists of the access interface to the telephone service provided by a TCO. In the signaling plane, it corresponds to the channel that carries the userside signaling to the telephone exchange (e.g., DTMF, Q.931 [23]), and in the voice plane, it corresponds to the channel that carries the analog signal or the coded digital voice samples.

The telephone layer is implemented in the telephone exchange. In the signaling plane, it includes the information exchanges with the user-side signaling agent and, consequently, the control of the telephone call (e.g., setup, tear down), obtained through network signaling mechanisms made avail- 


\begin{tabular}{|c|c|c|c|c|c|c|c|c|}
\hline \multirow{3}{*}{$\begin{array}{l}\text { SCENARIO } \\
\text { LAYER/ } \\
\text { INTERFACE }\end{array}$} & \multirow{2}{*}{\multicolumn{2}{|c|}{$\begin{array}{c}\text { IP Trunking } \\
\text { Scenario I }\end{array}$}} & \multicolumn{4}{|c|}{ IP Telephony } & \multirow{2}{*}{\multicolumn{2}{|c|}{$\begin{array}{c}\text { Internet Telephony } \\
\text { Scenario IV }\end{array}$}} \\
\hline & & & \multicolumn{2}{|c|}{ Scenario II (Legacy Access) } & \multicolumn{2}{|c|}{ Scenario III (IP access) } & & \\
\hline & $\begin{array}{l}\text { Signaling } \\
\text { Plane }\end{array}$ & Voice Plane & $\begin{array}{l}\text { Signaling } \\
\text { Plane }\end{array}$ & Voice Plane & $\begin{array}{l}\text { Signaling } \\
\text { Plane }\end{array}$ & Voice Plane & $\begin{array}{l}\text { Signaling } \\
\text { Plane }\end{array}$ & $\begin{array}{l}\text { Voice } \\
\text { Plane }\end{array}$ \\
\hline Access Interface & $\begin{array}{l}\text { User/Telephone } \\
\text { signaling } \\
\text { (e.g., E.164) }\end{array}$ & $\begin{array}{l}\text { User/Telephone } \\
\text { voice }\end{array}$ & $\begin{array}{l}\text { User/Telephone } \\
\text { signaling } \\
\text { (e.g., E.164) }\end{array}$ & $\begin{array}{l}\text { User/Telephone } \\
\text { voice }\end{array}$ & \begin{tabular}{|l|} 
User/IP \\
Telephone \\
signaling \\
(e.g., E.164, URI)
\end{tabular} & $\begin{array}{l}\text { User/IP } \\
\text { Telephone voice }\end{array}$ & \begin{tabular}{|l|} 
User/IP \\
Telephone \\
signaling \\
(e.g., E.164, URI)
\end{tabular} & $\begin{array}{l}\text { User/IP } \\
\text { Telephone voice }\end{array}$ \\
\hline Access & $\begin{array}{l}\text { PSTN/ISDN } \\
\text { signaling (user } \\
\text { side) }\end{array}$ & \begin{tabular}{|l|} 
PSTN/ISDN \\
voice \\
coding/decoding
\end{tabular} & $\begin{array}{l}\text { PSTN/ISDN } \\
\text { signaling (user } \\
\text { side) }\end{array}$ & \begin{tabular}{|l|} 
PSTN/ISDN \\
voice \\
coding/decoding
\end{tabular} & $\begin{array}{l}\text { VoIP signaling } \\
\text { (user side) }\end{array}$ & $\begin{array}{l}\text { VoIP } \\
\text { coding/decoding } \\
\text { packetization }\end{array}$ & $\begin{array}{l}\text { VoIP signaling } \\
\text { (user side) }\end{array}$ & $\begin{array}{l}\text { VoIP } \\
\text { coding/decoding } \\
\text { packetization }\end{array}$ \\
\hline $\begin{array}{l}\text { Telephone } \\
\text { Interface }\end{array}$ & \begin{tabular}{|l|} 
PSTN/ISDN \\
signaling (e.g., \\
DTMF, Q.931) \\
\end{tabular} & \begin{tabular}{|l} 
Analog or \\
digital Voice \\
(PCM G.711)
\end{tabular} & \begin{tabular}{|l|} 
PSTN/ISDN \\
signaling (e.g., \\
DTMF, Q.931) \\
\end{tabular} & \begin{tabular}{|l} 
Analog or \\
digital Voice \\
(PCM, G.711) \\
\end{tabular} & $\begin{array}{l}\text { VoIP signaling } \\
\text { (e.g., H.323, SIP) }\end{array}$ & $\begin{array}{l}\text { VoIP Coded } \\
\text { Voice } \\
\text { (e.g.G.723.1) } \\
\end{array}$ & $\begin{array}{l}\text { VoIP signaling } \\
\text { (e.g., H.323, SIP) }\end{array}$ & \begin{tabular}{|l|} 
VoIP Coded \\
Voice (e.g. \\
G.723.1) \\
\end{tabular} \\
\hline Telephone & $\begin{array}{l}\text { PSTN/ISDN } \\
\text { signaling } \\
\text { (network side) } \\
\text { Call Control }\end{array}$ & Voice transport & \begin{tabular}{|l|} 
Signaling \\
translation: \\
PSTN/ISDN to \\
VoIP \\
E.164 number \\
resolution \\
Network Access \\
Control
\end{tabular} & \begin{tabular}{|l|} 
Voice \\
conversion: \\
PSTN/ISDN to \\
VoIP \\
Voice transport \\
over RTP \\
Network Access \\
Filtering
\end{tabular} & $\begin{array}{l}\text { E.164 number / } \\
\text { URI resolution } \\
\text { Network Access } \\
\text { Control }\end{array}$ & $\begin{array}{l}\text { Voice transport } \\
\text { over RTP } \\
\text { Network Access } \\
\text { Filtering }\end{array}$ & $\begin{array}{l}\text { E.164 number / } \\
\text { URI resolution }\end{array}$ & $\begin{array}{l}\text { Voice transport } \\
\text { over RTP }\end{array}$ \\
\hline Network Interface & \begin{tabular}{|c|} 
Network \\
Signaling (e.g., \\
SS7) \\
\end{tabular} & $\begin{array}{l}\text { 64Kbps circuit } \\
\text { (e.g., DS0) }\end{array}$ & \multicolumn{4}{|c|}{ IP Interface over private IP Addresses } & \multicolumn{2}{|c|}{$\begin{array}{l}\text { IP Interface over public IP } \\
\text { Addresses }\end{array}$} \\
\hline Network & $\begin{array}{c}\text { Circuit } \\
\text { Establishment }\end{array}$ & TDM & TCO IP $N$ & Network for Telep & phony (public or $\mathrm{c}$ & corporate) & $\begin{array}{r}\text { ISP IP Network } \\
\text { Inte }\end{array}$ & $\begin{array}{l}\text { connected to the } \\
\text { rnet }\end{array}$ \\
\hline $\begin{array}{l}\text { Transmission } \\
\text { Layer Interface } \\
\end{array}$ & \multicolumn{2}{|c|}{ Circuit Emulation (T1/E1) } & \multicolumn{4}{|c|}{ Tunneling } & \multicolumn{2}{|c|}{ Tunneling } \\
\hline Transmission & \multicolumn{8}{|c|}{ IP Core Network } \\
\hline
\end{tabular}

able by the network layer. In the voice plane, it consists of the voice transport through switched circuits, and, optionally, of digital voice coding.

The network interface corresponds to the network signaling interface (e.g., SS7) in the signaling plane, while it corresponds to a 64-kb/s switched circuit endpoint (e.g., DS0) in the voice plane.

The network layer is implemented by the TCO network infrastructure (based on Class-4/5 switches). In the signaling plane, it corresponds to the setup of circuits, while in the voice plane, it corresponds to TDM-CS voice transport.

The transmission layer is implemented as an IP network. The transmission interface is based on "circuit emulation." SDH/PDH TDM lines (e.g., STS-1, T1/E1) are packetized and encapsulated in IP packets at the source and are reconstructed at the destination. The distinctive characteristic of this scenario is that the telephone network is implemented in the same way as in PSTN/ISDN, i.e., by connecting SDH/PDH lines to TDM-CS switches, while it is not influenced by the fact that the core network is based on IP. ${ }^{1}$

\footnotetext{
${ }^{1}$ An alternative solution consists of extracting each telephone call from T1 channels, converting it to a RTP flow and encapsulating the RTP flow in IP packets. The advantage of this solution is that voice is compressed with appropriate VoIP coding standards, so that the bandwidth requirements are reduced by up to one order of magnitude. This solution is a hybrid between IP trunking and IP telephony.
}

\section{B. Scenario II-IP Telephony With Legacy Access}

Scenario II shows the architecture of a TCO that adopts IP routing instead of TDM-CS at the network layer, while it provides the PSTN/ISDN interface.

The access interface, the access layer, and the telephone interface are equal to those of Scenario I.

The telephone layer, implemented in the telephone exchange, includes the following functions.

In the signaling plane:

1) Translation from user-side signaling (e.g., DTMF, Q.931) to VoIP signaling (e.g., H.323, SIP).

2) Translation of the telephone number into an IP address, using VoIP signaling. The IP address allows the IP-based network layer to route the call datagrams, provided that the destination IP address is reachable.

3) Network access control, through the configuration of appropriate network access firewalls, to grant the access only to those IP packets that carry a telephone call established through a correct signaling procedure.

In the voice plane:

1) translation from analog or digital (e.g., G.711) voice to formats specifically designed to code voice efficiently for PS (e.g., G.723.1[21]); 
Table 2

Synthesis of the Characteristics of IP-Supported Telephony Scenarios

\begin{tabular}{|c|c|c|c|c|c|}
\hline Scenario & IP feature & $\begin{array}{l}\text { Replaced } \\
\text { components }\end{array}$ & $\begin{array}{l}\text { Contrasted } \\
\text { Legacy }\end{array}$ & Added Value & VOIP \\
\hline I & $\begin{array}{l}\text { Statistical } \\
\text { Multiplexing }\end{array}$ & $\begin{array}{l}\text { SDH/PDH statically } \\
\text { structured frames }\end{array}$ & DT & $\begin{array}{l}\text { Dynamic bandwidth } \\
\text { allocation }\end{array}$ & $\mathrm{NO}$ \\
\hline II & $\begin{array}{l}\text { - } \begin{array}{c}\text { Statistical } \\
\text { Multiplexing }\end{array} \\
\text { - Packet } \\
\text { Switching }\end{array}$ & $\begin{array}{l}\text { SDH/PDH lines } \\
\text { - } \quad \text { statically } \\
\text { structured frames } \\
\text { - class-4/class5 } \\
\text { Switches and PBX } \\
\end{array}$ & $\begin{array}{l}\text { DT } \\
\text { DE }\end{array}$ & $\begin{array}{l}\text { - Dynamic bandwidth } \\
\text { Allocation } \\
\text { - Data Compression } \\
\text { - Equipment } \\
\text { Downsizing } \\
\end{array}$ & YES \\
\hline III & 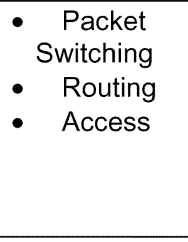 & $\begin{array}{lc}\text { - } & \text { SDH/PDH lines } \\
\text { - } & \text { class-4/class5 } \\
\text { Switches and PBX } \\
\text { - } \quad \text { (Terminals) }\end{array}$ & $\begin{array}{l}\text { DT } \\
\text { DE } \\
\text { (UI) }\end{array}$ & $\begin{array}{l}\text { - Dynamic bandwidth } \\
\text { Allocation } \\
\text { - Data compression } \\
\text { - Equipment } \\
\text { Downsizing } \\
\text { - Integrated } \\
\text { Data/Telephone access }\end{array}$ & YES \\
\hline IV & 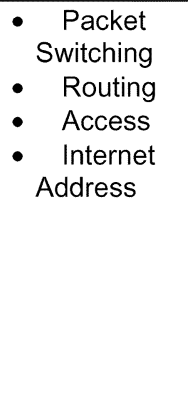 & $\begin{array}{l}\text { - } \mathrm{SDH} / \mathrm{PDH} \text { lines } \\
\text { - } \text { class-4/class5 } \\
\text { Switches and PBX } \\
\text { - (Terminals) } \\
\text { - TCO Telephone } \\
\text { Network }\end{array}$ & $\begin{array}{l}\text { DT } \\
\text { DE } \\
\text { CB } \\
\text { SL } \\
\text { (UI) } \\
\text { (TA) }\end{array}$ & $\begin{array}{l}\text { Dynamic bandwidth } \\
\text { Allocation } \\
\text { - Data compression } \\
\text { Equipment } \\
\text { Downsizing } \\
\text { Integrated } \\
\text { Data/Telephone access } \\
\text { Elimination of TCO } \\
\text { costs thanks to the use } \\
\text { of the Internet as a } \\
\text { transport infrastructure } \\
\text { for telephony }\end{array}$ & YES \\
\hline
\end{tabular}

2) transport of coded voice over protocols specifically designed to carry continuous media over IP, typically the RTP;

3) packet filtering according to the control parameters defined in item 3) of the telephone layer in the signaling plane.

The network layer consists of a simple IP network, and the network interface consists of standard IP interface.

The transmission layer implements the basic IP mechanisms in the same way as the network layer. If the network layer IP network and the transmission layer IP network coincide, then it makes no sense to define the interface between the two layers. This solution eliminates any distinction between the network layer and the transmission layer. If the two networks do not coincide, then the transmission layer interface can implemented by tunnels (e.g., using IPSec). The network layer IP packets are encapsulated in transmission layer IP packets or appropriately tagged for fast switching (e.g., using MPLS [39]).

It is worth noticing that both the network layer IP network and the transmission layer IP network, both in the case in which they coincide and in the case in which they are distinct, are managed as private networks, i.e., they follow a private IP numbering plan [37].

\section{Scenario III-IP Telephony With IP Access}

Scenario III is equal to Scenario II except for the fact that in Scenario III the access interface, the access layer, and the telephone interface are based on IP technology instead of telephone legacy technology.
The access interface consists of the UI of an IP telephone, which can be either a dedicated system that emulates a legacy telephone set or a software application that either emulates the telephone set interface or provides a richer UI. In the signaling plane, the access interface corresponds either to the 12-button keypad, ringing, busy/free tones, etc. (as in Scenarios I and II), or to an application menu in which appropriate URIs [2] (e.g., sip:max @ unipd.it) can be used instead of E.164 numbers. In the voice plane, the access interface corresponds to the speaker/microphone of an IP telephone or to the speaker/microphone of a PC.

The access layer is implemented in the IP telephone client. In the signaling plane, it includes the generation/processing of VoIP signaling messages (e.g., according to H.323 or SIP). In the voice plane, it consists of VoIP sampling/coding (e.g., G.723.1) and transmission over appropriate transport protocols (e.g., RTP).

It is worth noticing that the access interface and the access layer can be equivalently implemented in this scenario using a legacy telephone set connected to a system (called residential gateway) that translates the legacy signaling (e.g., DTMF) and voice data (e.g., analog voice) generated by the telephone set to VoIP signaling (e.g., H.323 or SIP) and VoIP coded and packet voice (e.g., G.723.1, RTP).

The telephone interface consists of the access interface to the telephone service provided by a TCO that bases its network on IP. In the signaling plane, it consists of the exchange of signaling packets (e.g., H.323/SIP), while in the voice plane, it consists of the exchange of voice packets (e.g., RTP). 
The telephone layer includes the same functions included in the telephone layer of Scenario II, except for the fact that no signaling translation takes place in the signaling plane and that no media conversion takes place in the voice plane, as the telephone interface receives and processes signaling and voice in VoIP format.

The underlying layers and interfaces are equal to those of Scenario II.

\section{Scenario IV-Internet Telephony}

Scenario IV is equal to Scenario III, except for the fact that in Scenario IV, the network layer is implemented by an IP network belonging to the Internet, while in Scenario III the network layer is implemented by a private IP network.

This difference, although not extremely significant under the technology point of view, is, on the contrary, crucial under the architecture point of view, as we will discuss in the following section.

The use of the Internet in the network layer leaves the other layers and interfaces unvaried with respect to the corresponding IP telephony scenario, except for the telephone layer, which does not control the access of the IP packet to the Internet. Controlling the access of telephone call packets (both signaling and voice) to the Internet would not make sense, considering that the Internet is by definition open to transport any IP packet with no limitation.

\section{E. Taxonomy of IP-Supported Telephony}

Each of the four scenarios presented above can be associated to a particular way of taking advantage of IP in telephony, as summarized in Table 2. The columns of Table 2 report the specific IP features exploited in each scenario, the components of PSTN/ISDN that can be replaced in each scenario thanks to the adoption of IP, the legacy dimensions which each scenario stands out against, the added value that the adoption of IP delivers in each scenario, and the fact that each scenario takes advantage of VoIP protocols and technology or not.

In Scenario I, IP is used at the transmission level to replace SDH/PDH circuits. In this case, IP is used only to support point-to-point links between pairs of TDM-CS switches or more in general to build a network of TDM-CS switches. Thanks to statistical multiplexing, IP provides more efficient bandwidth utilization than TDM-CS. The only legacy dimension Scenario I stands out against is DT, while the DE legacy dimension related to the most expensive equipment, i.e., the telephone switches, remains. This scenario does not take advantage of VoIP protocols and technology.

In Scenario II, IP is used at the network level. IP routers are used to route telephone calls, provided that private network boundaries are not traversed. In fact, the use of a private IP addressing scheme has as a consequence the fact that IP routing is not able to route datagrams across different networks, even in the case in which such networks are based on IP. A further added value with respect to Scenario I is the replacement of legacy telephone switches by low-cost IP switches/routers. Scenario II stands out against the DT and

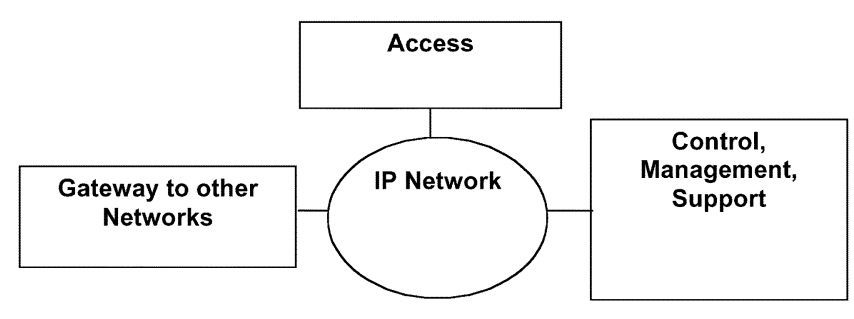

Fig. 2. VoIP-based telephony architecture.

the DE legacy dimensions. This scenario adopts VoIP protocols and technology.

In Scenario III, IP is used in the same way as in Scenario II. In addition it is also used at the access level, i.e., as a telephone service interface. The added valued in this case is that data and voice can be integrated, i.e., transported by the same protocol through the same access lines. Scenario III stands out against the DT and the DE legacy dimensions and potentially also against the UI legacy, even though the traditional UI of conventional telephone sets can still be maintained either by using IP telephones compliant with such an interface or by using traditional telephone sets hidden behind residential gateways. This scenario adopts VoIP protocols and technology.

In Scenario IV, IP, and more specifically the Internet, is used not only to replace the legacy network of a TCO but also to replace the entire global telephone network, taking advantage of the fact that the Internet is an existing global data network. Two Internet users can set up a end-to-end voice call in the same way as they can set up any other data transfer. In Scenario IV, the added value of implementing the network layer over the Internet rather than on a private IP network derives from the availability of a public address space (consisting of the public IP addresses and domain names) that allows addressing the users independently of telephone E.164 numbers.

The advantage of Scenario IV is that the telephone service, completely decoupled from the datagram transport service provided by the Internet, can be controlled by the users at the network endpoints by means of low-cost software applications. Scenario IV stands out against all the legacy dimensions. In particular, in addition to the legacy dimensions mentioned in the other scenarios, Scenario IV stands out against the SL legacy dimension, as the Internet is not able to guarantee SLs as PSTN/ISDN does. This scenario adopts VoIP protocols and technology.

\section{VoIP-Based Telephony ARChitecture}

This section focuses on the scenarios that take advantage of VoIP protocols and technology, namely the "IP telephony" scenarios (i.e., Scenarios II and III), in which IP is used in a TCO network, and the "Internet telephony" scenario (i.e., Scenario IV), in which the Internet is used as a telephone network. In particular, we show that these scenarios can be regarded as implementations of a common architecture, which can be taken as the architecture of VoIP-based telephony.

The elements of the architecture, shown in Fig. 2, are: 
Table 3

Implementation of the VoIP-Based Telephony Architecture

\begin{tabular}{l|l|l}
\hline Architecture Element & IP Telephony & Internet Telephony \\
\hline IP network & Managed IP Network with SLA & $\begin{array}{l}\text { Unmanaged IP Network with no } \\
\text { SLA }\end{array}$ \\
\hline Access Equipment & $\begin{array}{l}\text { IP access restricted by Media } \\
\text { Firewall }\end{array}$ & IP access (unrestricted) \\
\hline $\begin{array}{l}\text { Control Management and Support } \\
\text { Equipment }\end{array}$ & $\begin{array}{l}\text { Gatekeeper for: } \\
\text { Address Resolution } \\
\text { Access Control } \\
\text { General Service Management } \\
\text { (e.g., billing) }\end{array}$ & $\begin{array}{l}\text { Domain Name System with ENUM } \\
\text { Address Resolution }\end{array}$ \\
\hline Gateway to Other Network & $\begin{array}{l}\text { Telephone Call gateway } \\
\text { (application layer) with other } \\
\text { TCOs }\end{array}$ & $\begin{array}{l}\text { Best Effort IP router for peering } \\
\text { and/or transit }\end{array}$ \\
\hline
\end{tabular}

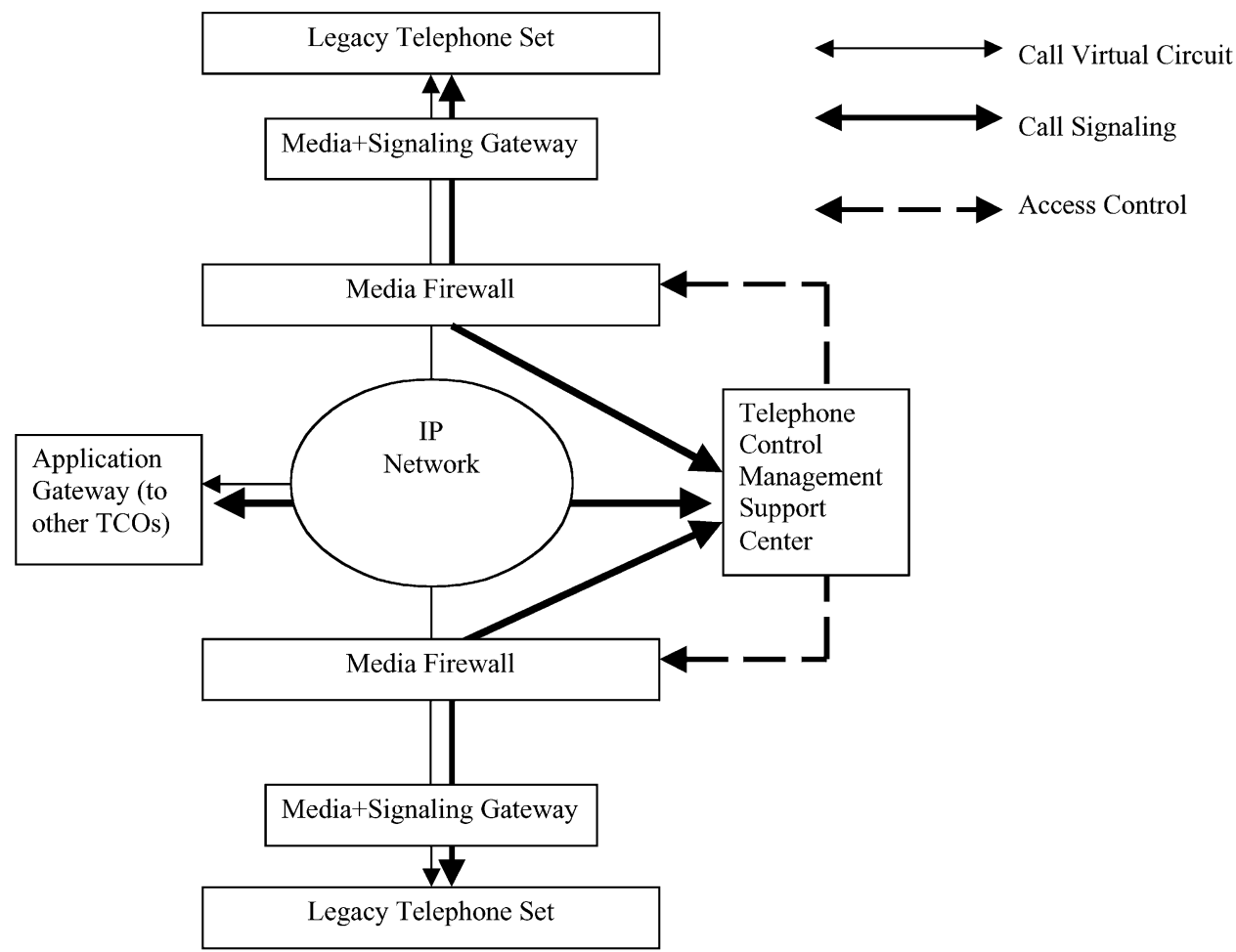

Fig. 3. IP telephony implementation of VoIP-based telephony architecture.

- IP network;

- access;

- control, management, and support;

- gateway for connection with other networks.

Each element is instantiated by different components, depending on whether IP telephony or Internet telephony is implemented, as indicated in Table 3.

In IP telephony (see Fig. 3), the architecture elements are instantiated by the following components.

- The "IP network" element corresponds to an IP network able to guarantee SLs. Considering that pure IP does not support SL guarantees, usually the transmission SLs are guaranteed by oversizing the network while the service availability levels are guaranteed by providing appropriate redundancy in the network resource.
- The "control, management, and support" element corresponds to a system that performs the following functions.

— Number resolution, i.e., translation from the E.164 telephone numbers managed by the TCO to the private IP addresses assigned to terminal and gateways by the TCO. This function is implemented by a module called Gatekeeper.

- Access control, i.e., handling of call establishment requests by instructing the media firewalls of the call endpoints to grant/deny network access to the call datagrams.

- General service management functions, including, for example, network monitoring and billing.

- The "gateway to other networks" element corresponds to application gateways that implement the interconnection with other TCOs call by call, at the telephone 


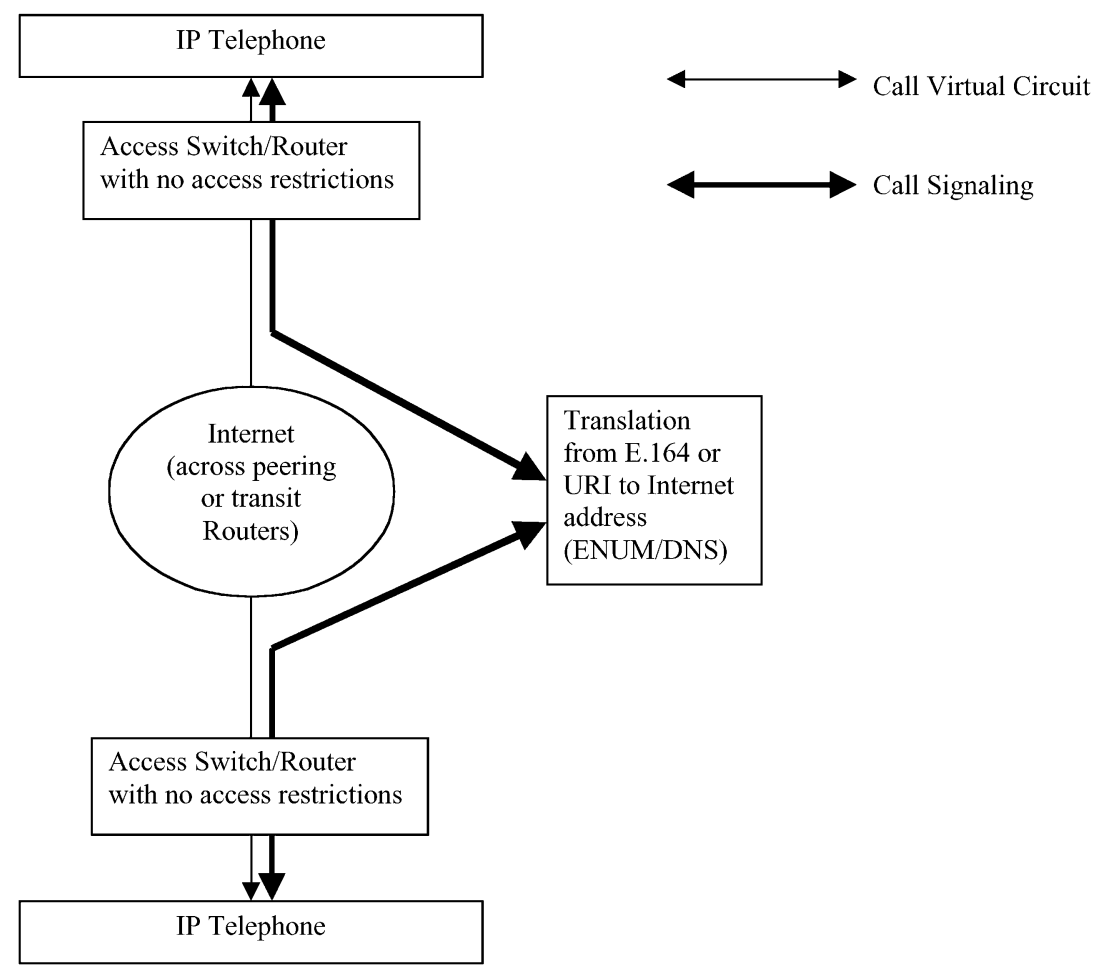

Fig. 4. Internet telephony implementation of VoIP-based telephony Architecture.

layer. Calls are exchanged between TCOs under specific commercial agreements, which need call level accounting and management.

- The "access" element corresponds to a set of firewalls that prevent the datagrams from entering the IP network unless they are part of authorized connections. Before injecting voice traffic in the TCO network, an IP telephone or a residential gateway signals the network, through the "telephone control, management, and support" element, its intention to establish a telephone call circuit. If the access is granted, then two permission rules are dynamically created in the media firewalls of the virtual circuit endpoints.

In Internet telephony (see Fig. 4), the architecture elements are instantiated by the following components.

- The "IP network" element corresponds to the ISP network and, through it, to the Internet, which guarantees no SL.

- The "access" element corresponds to a typical access switch/router with no access control mechanism, which allows any datagram to be injected into the Internet and travel with no regard to what it transports.

- The "telephone control, management, and support" element corresponds to a resolution mechanism that allows retrieving the IP address of the telephone call destination node. In those cases in which E.164 numbers are used for user identification, it is implemented as number resolution; in other words, it consists of the identification of the public IP addresses corresponding to an E.164 telephone number. The ENUM [12] system, currently under deployment on the Internet, implements this function. In those cases in which regular domain names are used for user identification, the existing DNS system implements the mapping of user identifiers to public IP addresses.

- The "gateway to other networks" element corresponds to the set of routers that support peering or transit between ISPs.

In summary, given the architecture in Fig. 2, the difference between IP telephony and Internet telephony derives from the different policy to grant/deny network access. While in Internet telephony, no limitation exists to Internet access, on the contrary, in IP telephony, the IP network is surrounded by a barrier of firewalls that block any traffic but that authorized by the telephone control, management, and support center. Any connection must be previously signaled to such an element.

\section{VOICE-DATA INTEGRATION ON DATA NETWORKS AND ON IP-SUPPORTED TELEPHONE NETWORKS}

One of the reasons that motivate the adoption of IP as a common protocol for voice and data transport is the possibility of supporting voice-data integration and, as a consequence, the possibility of implementing multimedia services based on such integration. However, voice-data integration and multimedia services do not automatically come as an effect of the adoption of IP, while on the contrary they require that appropriate connection schemes be applied at the user location. This section investigates these schemes in the light of the scenarios introduced in Section IV and of the architecture introduced in Section V. We consider the general case in which a user is connected both to the Internet, through an 


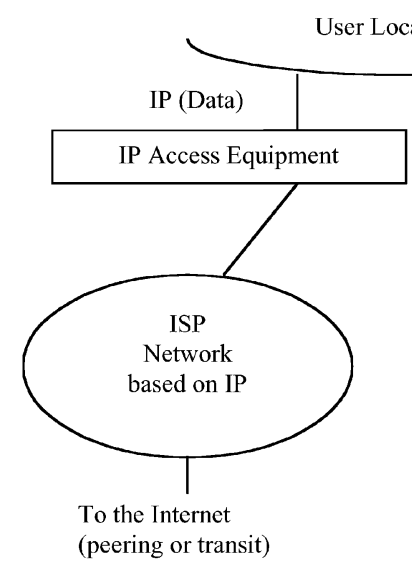

Fig. 5. IP trunking for PSTN support-no voice-data integration.

ISP, and to the telephone, through a TCO, and analyze how these two services can be integrated.

\section{A. Scheme 1: IP Trunking for PSTN/ISDN Support-No Voice-Data Integration}

Fig. 5 shows a scheme in which no voice-data integration takes place, as the user is connected to two different networks, one for Internet access and one for telephone access. The fact that the two networks are based on IP has no effect on voice-data integration because the characteristic of the telephone network of being based on IP is hidden to the user. The distinctive characteristics of this scheme are the following.

- Two separate networks exist, one for Internet access and one for telephone access.

- The data network, based on IP, is operated by an ISP and is connected to the Internet;

- The TCO network, based on TDM-CS, runs over an IP core network, is operated by a TCO, and is connected to the PSTN/ISDN;

- The two networks support different services; in particular, the ISP network supports Internet connectivity, whereas the TCO network supports the telephone service.

- Two access physical circuits exist, one for data, based on IP over a data link protocol (e.g., ATM, PPP), and one for voice, based on the PSTN/ISDN telephone interface. The two circuits are distinct at the physical layer, e.g., they may correspond to different wires or be multiplexed over the same wire, as in the case of xDSL. In this case, the two access equipment boxes can be thought of as one single access equipment box, able to separate the voice and data channels and connect each of them to the appropriate network.

- The user sees two different networks and, as a consequence, no integration may take place.

Scenario I of Section IV is the reference scenario for the TCO network in this scheme.

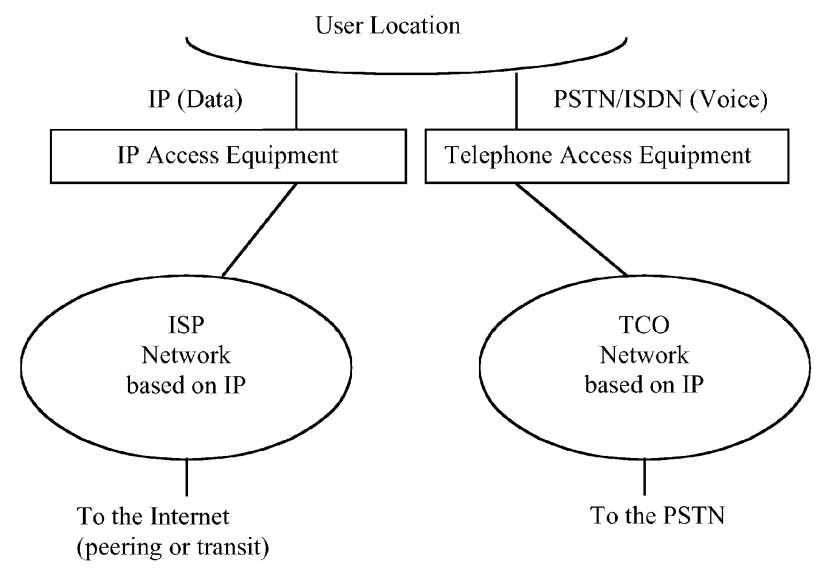

Fig. 6. IP telephony-No voice-data integration.

\section{B. Scheme 2: IP Telephony-No Voice-Data Integration}

Fig. 6 shows a scheme in which no voice-data integration takes place, as in Scheme 1. The user is connected to two different networks, one for Internet access and one for telephone access and, as in Scheme 1, the fact that the two networks are based on IP has no effect on voice-data integration because the characteristic of the telephone network of being based on IP is hidden to the user. The distinctive characteristics of this scheme are the following.

- Two distinct networks exist, one for Internet access and one for telephone access, as in Scheme 1. The difference with respect to Scheme 1 is that in Scheme 2 the TCO network is based on IP.

- Two access physical circuits exist, as in Scheme 1.

- The user sees two different networks and, as a consequence, no integration may take place, as in Scheme 1.

Scenario II of Section IV is the reference scenario for the TCO network in Scheme 2.

\section{Scheme 3: IP Telephony-Voice-Data Integration Within the TCO Network}

Fig. 7 shows a scheme in which voice-data integration takes place within the TCO network but not between the Internet and the TCO network. The TCO can provide its customers with advanced services (e.g., videoconference, video on demand, etc.) in addition to the telephone service, using the same private network for telephone and advanced services. As in Scheme 2, the user is connected to two different networks, one for Internet access, through the ISP network, and one for telephone and multimedia service access, through the TCO network. The distinctive characteristics of Scheme 3 are the following.

- Two distinct networks exist, as in Scheme 1 and in Scheme 2.

- The two networks support different services; in particular, the ISP network supports Internet access, while the TCO network supports the telephone service, connected to the PSTN, as well as additional multimedia services.

- While the ISP network is based on public Internet IP addresses and is connected to the Internet at the 


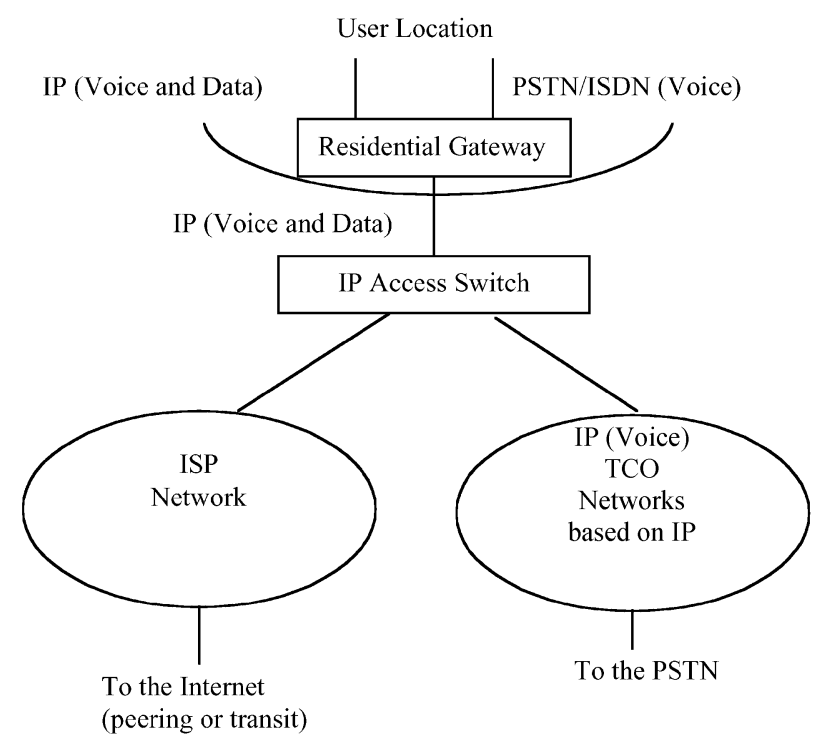

Fig. 7. IP telephony-voice-data integration in the TCO network.

IP level, the TCO network is based on private IP addresses and is not connected to the Internet.

- The access network is operated by a TCO or by company that plays the joint role of ISP and TCO, if the two services are provided by the same company; or, in the alternative, it is operated by a third company (usually the incumbent TCO operator), which separates the private traffic (telephone and additional multimedia data services) from the Internet traffic and brings the private traffic to the TCO and the Internet traffic to the ISP.

-One physical access circuit exists between the user location and the access operator point of presence. On the user side, such a circuit is terminated on a device called the residential gateway, while on the network side, it is terminated on the access equipment controlled by the ISP-TCO operator or by the company in charge of access operations.

- Two permanent virtual circuits exist, based, for example, on ATM technology: the first, for Internet access, between the user network to be connected to the Internet and the ISP, and the second, for telephone and additional multimedia data service access, between the user terminals where these services are to be delivered and the TCO.

-A device called the residential gateway is used to present the traditional telephone service interface (PSTN/ISDN) on the user side, in such a way to allow the user to interact with the traditional telephone service.

Scenario III of Section IV is the reference scenario for the TCO network in Scheme 3.

\section{Scheme 4: Internet Telephony-Voice-Data Integration Over the Internet}

Fig. 8 shows a scheme in which voice-data integration takes place over the Internet, which is used as a transport infrastructure also for telephone and additional multimedia ser-

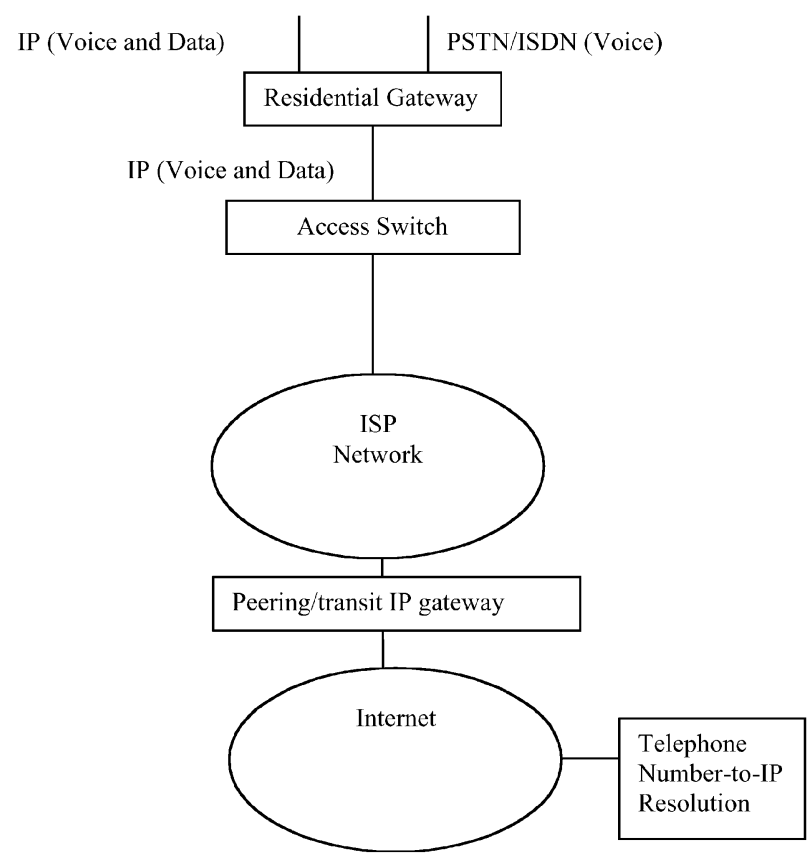

Fig. 8. Internet telephony-voice-data integration over the Internet.

vices. Unlike in the previous schemes, the user is connected to one network, i.e., the Internet, through the ISP network. All the services are supported by this network. The distinctive characteristics of Scheme 4 are the following.

- Only one network exists, namely, the ISP network, which is part of the Internet.

- The Internet supports connectionless datagram transport service, which is used by data and by telephone and multimedia services.

- Voice is transported over IP datagrams, either within the boundaries of the ISP network, if the communication endpoints are connected to the same ISP network, or over the Internet, if the communication endpoints are connected to the Internet through different ISP networks.

- The ISP network is managed by the ISP. No TCO is included in the scheme.

- Only one access circuit exists, between the user terminal and the ISP network point of presence.

- As in the previous scheme, a residential gateway may be used to present the traditional telephone service interface (PSTN/ISDN) on the user side, in such a way to allow the user to access the traditional telephone service.

Scenario IV of Section IV is the reference scenario for Scheme 4.

\section{E. Comparison of the Voice-Data Integration Schemes}

Schemes 1 and 2 do not support voice-data integration. In both Schemes 1 and 2, IP is adopted as a PS protocol by the TCO to reduce the implementation, operation, and management costs of its network by taking advantage of the low cost of IP equipment. The fact that IP instead of TDM-CS 
is used in the TCO network has no influence on the service provided to the user.

Schemes 3 and 4 deserve a deeper analysis. In Scheme 3, the TCO network is able to support not only the traditional telephone service but also additional multimedia services, such as, for example, pay TV, and data services, such as, for example, Internet access. All these services are managed so that they are delivered to the TCO customers under SL agreements and can be charged to the users according to the SLs provided. In Scheme 3, CPs make special agreements with TCOs in force of which they provide their services to the TCO customers at a given cost and at given SLs. The TCOs market the CP services, bill the users, and share the revenues with the CP. In Scheme 3, voice-data integration is achieved through an evolution of the TCO network toward an integrated voice-data network.

In Scheme 4, the telephone service, the multimedia services, and, more in general, all the application services are provided over the Internet, and the TCO network has no role. CPs interact directly with the application service users, with no intermediary. Voice-data integration is achieved through an evolution of the Internet toward an integrated voice-data network.

\section{DISCUSSION}

The adoption of IP as a PS protocol to support telephony, usually associated to the VoIP acronym, is often perceived as a step toward the integration between telephony and Internet. This perception does not necessarily correspond to reality, as the mere adoption of IP as a PS protocol does not have as a direct consequence a shift from the telephone philosophy to the Internet philosophy.

As we have shown in Section II, the Internet philosophy can be summarized through a set of principles that are substantially different from the principles of telephone philosophy. While it is the application of the Internet philosophy principles that led in the past to the definition of the IP protocol, it is, however, possible to adopt only the IP protocol without adhering to the Internet philosophy. In this case, IP is nothing more than a communication model (unacknowledged datagram) and a packet format (the IP packet) widely implemented in network equipment (hosts, routers, etc.).

We review here the reference model introduced in Section IV to identify which scenarios of IP-supported telephony bring only the IP to the telephony and which scenarios bring both the IP and the Internet philosophy to the telephony, as synthesized by Table 4 .

As observed in Section IV, Scenario I, which corresponds to the use of IP to support virtual circuits, does not belong to what is usually called VoIP, as voice is mapped on IP indirectly, through a traditional telephone network. In this case, the network uses circuits emulated over an IP network, through an appropriate transport protocol, in the same way as it might use physical circuits or MPLS/ATM/frame relay virtual circuits. Scenario I brings the IP protocol in the telephone system but does not follow the Internet philosophy.
Table 4

IP-Supported Telephony Philosophies

\begin{tabular}{l|l|l}
\hline Scenario & Name & Philosophy \\
\hline Scenario I & IP Trunking & Telephone \\
\hline Scenario II, III & IP Telephony & Telephone \\
\hline Scenario IV & Internet Telephony & Internet \\
\hline
\end{tabular}

On the contrary, as observed in Section IV, Scenarios II, III, and IV of IP-supported telephony belong to VoIP. Scenarios II and III, called IP telephony, are the ones in which a TCO adopts IP as a PS protocol to improve its efficiency and to support the integration of voice and data in multimedia services. Scenario IV, called Internet telephony, is the one in which the Internet is used to transport telephone conversations. While IP telephony, in spite of the adoption of IP, is based on the telephone philosophy, Internet telephony is based on the Internet philosophy.

The distinctive features of Internet telephony, with respect to IP telephony can be summarized as follows.

1) The use of an IP network that does not provide SL guarantees, i.e., the ISP network and, through it, the Internet. On the contrary, in IP telephony the IP network is under the control of the TCOs and provides SL guarantees.

2) The use of the Internet IP public address space, which allows taking advantage of the global nature of the Internet and, as a consequence, to establish telephone conversation with any other terminal connected to the Internet. On the contrary, in IP telephony the IP addresses associated to the terminals belong to the TCO private address space, which has as a consequence the fact that telephone conversations between terminals connected to different telephone networks must be supported by call level gateways.

3) The adoption of an approach in which the network provides only the basic datagram transport service while all the application services, for call control and management and for multimedia integration, are implemented at the network periphery, either between the communication end points or by application service providers that do not necessarily act also as network service providers. On the contrary, in IP telephony the application services related to call control and management and to multimedia integration are implemented by the TCOs, which play the role of application service providers.

While the last two features can be considered in favor of Internet telephony with respect to IP telephony, the first feature, on the contrary, appears as a weak point of the Internet telephony. For this reason, we focus our analysis on the SLs, and in particular on the following issues:

1) whether the impossibility to guarantee SLs prevents the Internet from being considered suitable for voice transport; 
2) whether the present SL of the PSTN is to be taken as a reference and achieved by Internet telephony.

As far as the first issue is concerned, it is certainly true that the best effort nature of the Internet would seem to discourage the use of the Internet as a voice transport network, considering that best effort and SL guarantees are not compatible. However, if we consider statistical guarantees instead of hard guarantees, the above statement is not necessarily true. The questions are whether the Internet is able to transport intelligible telephone traffic, given its current capacity and its current traffic load, including both voice and data, and, in case of a positive answer, whether the Internet capacity evolves at least at the same rate as the rate at wich such a traffic load grows. The answer to the first question is positive, i.e., the total telephone traffic transported by the PSTN is currently of the same order of magnitude as the data traffic transported by the Internet, as shown in Section II. In addition, this latter traffic grows at a rate of about $70 \%$ per year [36] and is soon destined to become the dominant traffic. The answer to the second question is also positive, as we have seen that the ISPs tend to adapt the capacity of their infrastructures to the traffic level rapidly, so as to deliver an acceptable quality of service to their users.

As far as the second issue is concerned and with specific reference to service availability, the well known $99.999 \%$ factor is not implicit in the nature of telephone service, while on the contrary it is a legacy of the telephone service, as shown in Section III. This is demonstrated by the fact that after the liberalization of telecommunication services, some of the new operators (i.e., the OLOs) do not guarantee $99.999 \%$ service availability as the incumbents operators usually do. The rapid growth of the OLO CB demonstrates that a reduction of service availability is acceptable if it comes along with a drastic reduction of the service price.

However, the reason why at the present time Internet telephony can only be considered an additional service in addition to traditional telephony, be it based on IP or not, is that the TCOs provide a set of additional services in support to communication services that nobody provides in Internet telephony. These services, which are often considered implicit by the telephone users, are those that make of telephony an easily accessible service for all the people categories. We refer, for example, to installation, repair, maintenance, operator assistance, and so forth. The existence of these services and the impossibility to distinguish them from the basic telephone service is an instance of the CB legacy.

\section{CONCLUSION}

We have analyzed the evolution of the telephone service networks from TDM-CS technologies to IP-supported telephony. We have first presented, as a background, a comparison between the telephone philosophy and the Internet philosophy and a review of the legacy issues related to the telephone service. We have presented the design solutions associated to the evolution from TDM-CS to IP as follows. We have first introduced a reference model consisting of a set of layers associated to the different functions to be performed to support telephony and have then used such a reference model to establish a terminology and a framework for the comparison of the different solutions. We have grouped the different solutions in scenarios and have compared and discussed such scenarios in terms of the reference model proposed. We have then focused on IP telephony and Internet telephony and have shown that they both can be seen as implementations of the same architecture. We have finally considered the issue of voice-data integration and have analyzed the variety of design solutions that can be adopted to integrate voice and data.

\section{ACKNOWLEDGMENT}

The authors would like to thank Dr. D. Tezza for his help in tuning the manuscript and his suggestions.

\section{REFERENCES}

[1] J. G. Beerends et al., "Perceptual evaluation of speech quality (PESQ)-The new ITU standard for end-to-end speech quality assessment-Part II-Psychoacoust model," J. Audio Eng. Soc., vol. 50, no. 10, pp. 765-778, Oct. 2002.

[2] T. Berners-Lee, R. Fielding, and L. Masinter. (1998) Request for comments 2396: Uniform resource identifiers (URI): Generic syntax. [Online]. Available: http://www.ietf.org/rfc/rfc2396.txt

[3] P. Blatherwick, R. Bell, and P. Holland. (2001) Request for comments 3054: Megaco IP phone media gateway application profile. [Online]. Available: http://www.ietf.org/rfc/rfc3054.txt

[4] S. Bradner, "Internet telephony: Progress along the road," IEEE Internet Computing, vol. 6, no. 3, pp. 37-38, May-June 2002.

[5] R. Bush and D. Meyer. (2002) Request for comments 3439: Some Internet architectural guidelines and philosophy. [Online]. Available: http://www.ietf.org/rfc/rfc3439.txt

[6] “This week@EMEA (Cisco government affairs e-update)," Cisco Systems, San Jose, CA, vol. 2, Oct. 2002.

[7] K. G. Coffman and A. M. Odlyzko, "Growth of the Internet," in Optical Fiber Telecommunications IV-B: Systems and Impairments. New York: Academic, 2002, pp. 17-56.

[8] D. Collins, Carrier Grade Voice Over IP. New York: McGrawHill, 2000.

[9] D. S. Isenberg, "The dawn of the stupid network," in ACM Networker 2.1, vol. 2, Feb./Mar. 1998, pp. 24-31.

[10] H. J. Engelbrecht, "Statistics for the information age," Inf. Econ. Policy, vol. 13, no. 3, pp. 339-349, Sept. 2001.

[11] "European digital cellular telecommunications system (phase 2): Full rate speech transcoding (GSM 06.10)," ETSI, Sophia Antipolis, France, Tech. Rep. ETS 300 580-2, 1994.

[12] P. Faltstrom. (2000) Request for comments 2916: E. 164 number and DNS. [Online]. Available: http://www.ietf.org/rfc/rfc2916.txt

[13] GARR - The Italian Academic and Research Network (2001). [Online]. Available: http://www.garr.it/garr-b-home-engl.shtml

[14] J. Garrahan, P. Russo, K. Kitami, and R. Kung, "Intelligent network overview," IEEE Commun. Mag., vol. 31, pp. 30-37, Mar. 1993.

[15] M. Gaynor, "Linking market uncertainty to VoIP service architectures," IEEE Internet Comput., vol. 7, pp. 16-22, July-Aug. 2003.

[16] B. Goode, "Voice over Internet Protocol (VoIP)," in Proc. IEEE, vol. 90, Sept. 2002, pp. 1495-1517.

[17] N. Greene, M. Ramalho, and B. Rosen. (2000) Request for comments 2805: Media gateway control protocol architecture and requirements. [Online]. Available: http://www.ietf.org/rfc/rfc2805.txt

[18] J. Rosenberg, H. Schulzrinne, G. Camarillo, A. Johnston, J. Peterson, R. Sparks, M. Handley, and E. Schooler. (2002) Request for comments 3261: SIP: Session initiation protocol. [Online]. Available: http://www.ietf.org/rfc/rfc3261.txt

[19] H. J. Helgert, Integrated Services Digital Networks, Architectures, Protocols and Standards. Reading, MA: Addison-Wesley, 1991.

[20] "Packet based multimedia communication systems version 5," ITU Telecommunication Standardization Sector (ITU-T), Geneva, Switzerland, ITU-T Recommendation H.323, July 2003. 
[21] "Dual rate speech coder for multimedia communication transmitting at 5.3 and $6.3 \mathrm{kbit} / \mathrm{s}$," ITU Telecommunication Standardization Sector (ITU-T), Geneva, Switzerland, ITU-T Recommendation G.723.1, Mar. 1996.

[22] "The international public telecommunication numbering plan," ITU Telecommunication Standardization Sector (ITU-T), Geneva, Switzerland, ITU-T Recommendation E.164, May 1997.

[23] "Digital subscriber signaling system no. 1 (DSS1) - ISDN user-network interface layer 3 specification for basic call control," ITU Telecommunication Standardization Sector (ITU-T), Geneva, Switzerland, ITU-T Recommendation Q.931, 1993.

[24] "Digital hierarchy bit rates," ITU Telecommunication Standardization Sector (ITU-T), Geneva, Switzerland, ITU-T Recommendation G.702, Nov. 1988.

[25] "Network node interface for the synchronous digital hierarchy (SDH)/Sub STM-0 network node interface for the SDH/Interfaces for the optical transport network (OTN)," ITU Telecommunication Standardization Sector (ITU-T), Geneva, Switzerland, ITU-T Recommendations G.707/G.708/G.709, 1998/1999/2001.

[26] “Signaling system no. 7 (SS7)," ITU Telecommunication Standardization Sector (ITU-T), Geneva, Switzerland, ITU-T Recommendations Q.700-Q.795, CCITT Blue Book, 1988.

[27] Pulse Code Modulation (PCM) of Voice Frequencies, Nov. 1988. ITU-T, Recommendation G.711.

[28] S. Kent and R. Atkinson. (1998) Request for comments 2401: Security architecture for the IP protocol. [Online]. Available: http://www.ietf.org/rfc/rfc2401.txt

[29] D. R. Kuhn, "Sources of failure in the public switched telephone network," IEEE Computer, vol. 30, pp. 31-36, Apr. 1997.

[30] D. Lovell, Cisco IP Telephony. Indianapolis, IN: Cisco Press, 2001.

[31] A. P. Markopoulou, F. A. Tobagi, and M. J. Karam, "Assessing the quality of voice communications over Internet backbones," IEEE/ACM Trans. Networking, vol. 11, pp. 747-760, Oct. 2003.

[32] C. Mendler. (2003) Telecom Italia packet voice: A case study. Yankee Group, Boston, MA. [Online]. Available: http://newsroom. cisco.com/dlls/Telecom_Italia.pdf

[33] S. Nocentini and M. Siviero, "Innovative class 5: A challenge for incumbent network operators," J. Commun. Netw., pt. 2, vol. 1, pp. 52-55, July-Sept. 2002.

[34] A. M. Noll, "Does data traffic exceed voice traffic?," Commun. ACM, vol. 46, pp. 121-124, June 1999.

[35] J. Postel. (1981) Request for comments 791: Internet Protocol. [Online]. Available: http://www.ietf.org/rfc/rfc791.txt

[36] Global Internet Geography. Washington, DC: Primetrica, 2003.

[37] Y. Rekthter, B. Moskowitz, D. Karrenberg, G. J. de Groot, and E. Lear. (1996) Request for comments 1918: Address allocation for private Internets. [Online]. Available: http://www.ietf.org/rfc/rfc1918.txt

[38] A. W. Rix et al., "Perceptual evaluation of speech quality (PESQ) - The new ITU standard for end-to-end speech quality assessment-Part I-Time-delay compensation," J. Audio Eng. Soc., vol. 50, no. 10, pp. 755-764, Oct. 2002.

[39] E. Rosen, A. Viswanathan, and R. Callon. (2001) Request for comments 3031: Multiprotocol label switching architecture. [Online]. Available: http://www.ietf.org/rfc/rfc3031.txt

[40] M. V. Russo, "Technology, deregulation, and the public interest: Preserving universal telephone service," Technol. Soc. Magazine, vol. 7, no. 1, Mar. 1988

[41] H. Schultzrinne, S. Casner, R. Frederick, and V. Jacobson. (1996) Request for comments 1889: RTP: A transport protocol for real-time applications. [Online]. Available: http://www.ietf.org/rfc/rfc1889.txt

[42] G. A. Thom, "H.323: The multimedia communications standard for local area networks," IEEE Commun. Mag., vol. 34, pp. 52-56, Dec. 1996.

[43] M. Weiss and J. S. Shin, "Internet interconnection economic model and its analysis: Peering and settlement," presented at the 17th World Computer Congr., Montreal, QC, Canada, 2002.

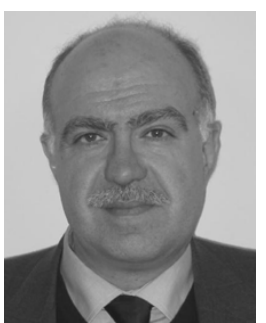

Massimo Maresca was born in Genova, Italy, in 1956. He received the Laurea degreee in electrical engineering and a Research Doctorate in computer engineering from the University of Genova, Genova, in 1980 and 1986, respectively.

From 1985 to 1986, he was a Postdoctoral Research Scientist at the IBM T. J. Watson Research Center, Yorktown Heights, NY, and, from 1989 to 1990 , at the International Computer Science Institute, Berkeley, CA. He took a tenured position as an Assistant Professor at the University of Genova in 1990. In 1992, he was promoted to the post of Associate Professor at the University of Genova. From 1992 to 1995, he also led the research unit of the University of Genova in the European ESPRIT Basic Research Project named "SMIMP_SIMD/MIMD architectures for image processing." In 1994 he joined the University of Padova, Padova, Italy, as a Professor. From 1999 to 2002, he also led the research unit of the University of Genoa in the Italian project named "CCS - the Cargo Community System."He is currently a Professor of Computer Engineering in the Department of Information Engineering, University of Padova. He is also the Coordinator of the European FP6 Project named "VISIONS—Vehicular Information System Interface for Open Network Services." In addition, he is the Scientific Supervisor of a research project funded by Marconi Communications in the area of IP telephony. He has published several papers in refereed journals, books, and conference proceedings. His research interests are in the area of parallel/distributed computing and networking. He started from massively parallel computer architectures, moved to instruction-level parallel architectures, and is currently working prevalently in the area of computer networks. In particular, he is exploring two application domains, namely, multimedia services and transport/logistics.

Dr. Maresca served two years (1998 and 1999) as a Chief Technical Adviser of the President of L'Autorità per l'Informatica nella Pubblica Amministrazione (AIPA), an Italian independent authority in charge of the deployment of computer and telecommunication technologies in the Italian public administration, and three years (1999-2001) as the Italian representative in the Governmental Advisory Committee (GAC) of the Internet Corporation for Assigned Names and Numbers (ICANN). He has also served on the program committees of several scientific workshops and conferences. He is currently on the Editorial Board of the PROCEEDINGS OF THE IEEE.

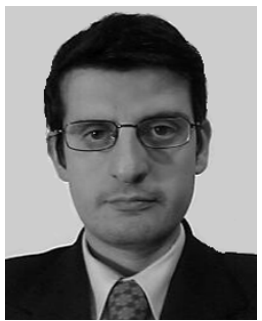

Nicola Zingirian was born in 1970. He received the Laurea degree from the University of Genova, Genova, Italy, in 1994 and the Ph.D. degree from the University of Padova, Padova, Italy, in 1998.

From 1999 to 2002, he was an Assistant Professor at the University of Padova. He is currently associate professor with the same university. His research interests include computer architectures-in particular, optimization techniques to improve the efficiency of computing intensive program executed on instruction level parallel processors-and computer network architectures-in particular, network architectures for IP telephony and tools for passive network monitoring.

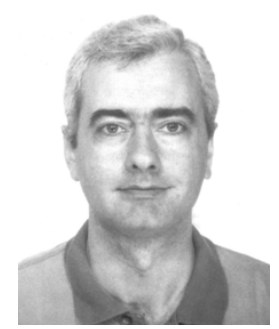

Pierpaolo Baglietto (Member, IEEE) was born in Varazze, Italy, in 1963. He received the Laurea and $\mathrm{Ph} . \mathrm{D}$. degrees from the University of Genova, Genova, Italy, in 1990 and 1994, respectively.

Fron 1996 to 2003, he was an Assistant Professor at the University of Genova, where he is currently an Associate Professor. His research interests are mainly in the areas of parallel/distributed computing and of network architectures and security. While his earlier works are in the field of massively parallel computer architectures and programming models, he is currently working in the area of network architectures for multimedia services. He is also involved in several research and technology transfer projects in the area of information and communication technologies applied to transport and logistic services. 